\title{
CONTRIBUCIÓN AL CONOCIMIENTO DE LAS AVES EN HOYO DE PELEMPITO, SIERRA DE BAHORUCO, REPÚBLICA DOMINICANA
}

\author{
Hodali Almonte-Espinosa \\ Museo Nacional de Historia Natural. "Prof. Eugenio de Jesús Marcano”. Calle César Nicolás Penson, \\ Plaza de la Cultura Juan Pablo Duarte, Santo Domingo, República Dominicana. \\ h.almonte@mnhn.gov.do
}

\section{RESUMEN}

Se realizó un inventario de las especies de aves presentes en el Hoyo de Pelempito, Sierra de Bahoruco. Mediante el uso de puntos de conteo se registró un total de 991 individuos, 41 especies y 17 familias de aves, encontrándose que 13 especies son endémicas, 18 residentes, nueve migratorias y dos introducidas. Seis especies se consideran amenazadas, entre estas se pueden citar el caso de la Cotorra (Amazona ventralis) y el Perico (Psittacara chloroptera). Las aves insectívoras y omnívoras fueron las más abundantes, mientras que las nectarívoras, granívoras, carnívoras y frugívoras se presentaron en menor proporción.

Palabras clave: Hoyo de Pelempito, comunidad de aves, riqueza de especies, gremios alimentarios, especies amenazadas.

\section{ABSTRACT}

An inventory of birds was performed at Hoyo de Pelempito, Sierra de Bahoruco. A total of 991 individuals, 41 species and 17 families of birds were recorded by using points count. Nine species are migratory, 13 endemic, 18 residents and two introduced. Six species are considered threatened; such as the Hispaniolan Parrot (Amazona ventralis) and Hispaniolan Parakeet (Psittacara chloroptera). Insectivorous and omnivores birds were the most abundant, while granivorous, nectarivores, carnivores and frugivorous occurred in a lesser extent.

Keywords: Hoyo de Pelempito, bird community, species richness, foraging guilds, threatened species.

La Hispaniola alberga unas 306 especies de aves, 136 de éstas han sido reportadas para la Sierra de Bahoruco, uno de los centros de mayor diversidad de la isla (Perdomo et al. 2010). Esta diversidad probablemente esté relacionada con su ubicación geográfica y la gran variedad microclimática que ofrecen los bosques de la zona.

Dentro de la gran multiplicidad de ambientes de la Sierra de Bahoruco se encuentra el Hoyo de Pelempito, el cual es una depresión tectónica ubicada a una altura de 348 metros sobre el nivel del mar (msnm), posee una extensión de aproximadamente $8 \mathrm{~km}^{2}$. Esta zona puede considerarse como una sabana natural con sustrato de arcilla y limo impermeables (Martínez Batlle, 2002), condición que favorece su inundación durante la época lluviosa. El tipo de vegetación presente en esta área corresponde al bosque seco, con una apreciable variedad florística. Favoreciendo la presencia de algunas especies de aves.

Los resultados que se presentan fueron obtenidos mediante el uso de puntos de conteos (Ralph et al., 1996). Además se utilizó el método de registro no sistemático de aves (Fjeldsa, 1999). Para la identificación de las especies se siguió a Latta et al. (2006) y para la taxonomía se 
han considerado diferentes suplementos de The American Ornithologist Union, AOU (Banks et al., 2005, 2006, 2007, 2008; Chesser et al., 2009, 2010, 2014). Se registraron 991 ejemplares, y 41 especies de aves agrupadas en 17 familias (Tabla I). Las familias mejor representadas fueron Parulidae, con diez especies, y Columbidae, con cinco, siendo esta última la más abundante.

Del total de especies registradas, 13 son endémicas, 18 residentes, nueve migratorias y dos introducidas (Tabla I). Las especies más abundantes fueron el Barrancolí (Todus subulatus), el Cuatro Ojos (Phaenicophilus palmarum) y la Cotorra (Amazona ventralis).

Tabla I. Especies de aves registradas en el Hoyo de Pelempito. $\mathrm{R}=$ Residente; $\mathrm{E}=$ Endémica; $\mathrm{I}=$ Introducida; $\mathrm{M}=$ Migratorias; $*=$ Especie amenazada. $\mathrm{C}=$ Carnívora, $\mathrm{O}=$ Omnívora, $\mathrm{G}-\mathrm{F}=$ Granívoro-Frugívoro, $\mathrm{G}=$ Granívora, $\mathrm{N}=$ Nectarívora, $\mathrm{F}=$ frugívoro.

\begin{tabular}{|c|c|c|c|c|c|}
\hline Familia & Especie & Nombre común & Estatus & $\begin{array}{c}\text { Gremio } \\
\text { alimentario }\end{array}$ & $\mathrm{N}^{\mathrm{o}}$ registros \\
\hline \multirow[t]{2}{*}{ Accipitridae } & Accipiter striatus* & Guaraguaíto de Sierra & $\mathrm{R}$ & $\mathrm{C}$ & 3 \\
\hline & Buteo jamaicensis & Guaraguao & $\mathrm{R}$ & $\mathrm{C}$ & 1 \\
\hline \multirow{5}{*}{ Columbidae } & Geotrygon chrysia* & Perdíz Grande & $\mathrm{R}$ & G & 65 \\
\hline & Columbina passerina & Rolita & $\mathrm{R}$ & G & 49 \\
\hline & Zenaida aurita & Rolón Turco & $\mathrm{R}$ & G & 7 \\
\hline & Zenaida asiática & Tortola Aliblanca & $\mathrm{R}$ & G-F & 16 \\
\hline & Patagioenas leucocephala* & Paloma Coronita & $\mathrm{R}$ & $\mathrm{F}$ & 22 \\
\hline \multirow{3}{*}{ Psittacidae } & Amazona ventralis * & Cotorra & $\mathrm{E}$ & G-F & 85 \\
\hline & Psittacara chloropterus * & Perico & $\mathrm{E}$ & G-F & 46 \\
\hline & Eupsittula nana & Perico Amargo & $\mathrm{I} ?$ & G-F & 25 \\
\hline \multirow{3}{*}{ Cuculidae } & Crotophaga ani & Judío & $\mathrm{R}$ & $\mathrm{O}$ & 12 \\
\hline & Coccyzus longirostris & Pájaro Bobo & $\mathrm{E}$ & $\mathrm{O}$ & 37 \\
\hline & Coccyzus minor & Pájaro Bobo Menor & $\mathrm{R}$ & $\mathrm{O}$ & 4 \\
\hline Tytonidae & Tyto glaucops & Lechuza Cara Ceniza & $\mathrm{E}$ & $\mathrm{C}$ & 2 \\
\hline \multirow{2}{*}{ Trochilidae } & Anthracothorax dominicus & Zumbador Grade & $\mathrm{R}$ & $\mathrm{N}$ & 12 \\
\hline & Mellisuga minima & Zumbadorcito & $\mathrm{R}$ & $\mathrm{N}$ & 3 \\
\hline \multirow{2}{*}{ Todidae } & Todus subulatus & Barrancolí & $\mathrm{E}$ & I & 96 \\
\hline & Todus angustirostris & Chi-cuí & $\mathrm{E}$ & I & 5 \\
\hline \multirow{2}{*}{ Picidae } & Melanerpes striatus & Carpintero & $\mathrm{E}$ & I & 54 \\
\hline & Nesoctites micromegas & Carpintero de Sierra & $\mathrm{E}$ & I & 32 \\
\hline \multirow{2}{*}{ Tyrannidae } & Myiarchus stolidus & Manuelito & $\mathrm{R}$ & I & 35 \\
\hline & Contopus hispaniolensis & Maroita & $\mathrm{E}$ & $\mathrm{I}$ & 52 \\
\hline \multirow{2}{*}{ Vireonidae } & Vireo altiloquus & Julian Chiví & $\mathrm{R}$ & I & 26 \\
\hline & Vireo nanus & Cigüita Juliana & $\mathrm{E}$ & I & 3 \\
\hline Corvidae & Corvus palmarum $*$ & $\mathrm{Cao}$ & $\mathrm{E}$ & $\mathrm{O}$ & 8 \\
\hline Turdidae & Turdus Plumbeus & Chua-chuá & $\mathrm{R}$ & $\mathrm{O}$ & 19 \\
\hline Mimidae & Mimus polyglottos & Ruiseñor & $\mathrm{R}$ & $\mathrm{O}$ & 22 \\
\hline
\end{tabular}




\begin{tabular}{|c|c|c|c|c|c|}
\hline Familia & Especie & Nombre común & Estatus & $\begin{array}{c}\text { Gremio } \\
\text { alimentario }\end{array}$ & $\mathrm{N}^{\circ}$ registros \\
\hline \multirow{10}{*}{ Parulidae } & Seiurus aurocapilla & Cigüita Saltarina & $\mathrm{M}$ & I & 9 \\
\hline & Mniotilta varia & Pegapalo & M & I & 7 \\
\hline & Setophaga caerulescens & Cigüita Azul & M & I & 9 \\
\hline & Setophaga ruticilla & Candelita & M & I & 10 \\
\hline & Setophaga magnolia & Cigüita Magnolia & M & I & 2 \\
\hline & Setophaga discolor & Cigüita de los Prados & M & I & 5 \\
\hline & Setophaga tigrina & Cigüita Tigrina & M & $\mathrm{I}$ & 6 \\
\hline & Setophaga parula & Cigüita Parula & $\mathrm{M}$ & I & 2 \\
\hline & Setophaga citrina & Cigüita Gorra Negra & $\mathrm{M}$ & I & 2 \\
\hline & Microligea palustris & Cigüita Cola Verde & $\mathrm{E}$ & I & 29 \\
\hline \multirow{3}{*}{ Thraupidae } & Coereba flaveola & Cigüita Común & $\mathrm{R}$ & $\mathrm{N}$ & 51 \\
\hline & Phaenicophilus palmarum & Cuatro Ojos & $\mathrm{E}$ & I & 74 \\
\hline & Loxigilla violácea & Gallito Prieto & $\mathrm{R}$ & G-F & 18 \\
\hline Numididae & Numida meleagris & Guinea & I & $\mathrm{O}$ & 26 \\
\hline 17 & 41 & & & & 991 \\
\hline
\end{tabular}

? Eupsittula nana. En este trabajo, se considera esta especie como introducida, sin embargo, se coloca un signo de interrogación debido a que se desconoce con exactitud la forma en que fue introducida en la isla.

El total de especies registradas constituye más del 30\% de las aves reportadas para el Parque Nacional Sierra de Bahoruco, donde han sido documentadas 136 especies (Perdomo et al., 2010). Además, en esta zona se encuentran 13 de las 31 especies endémicas registradas para la Hispaniola. A su vez, se encuentran seis especies consideradas bajo alguna categoría de amenaza según la Lista Roja de la Unión Mundial para la Naturaleza UICN: la Cotorra (A. ventralis), el Perico (P. chloropterus), la Perdiz Grande (G. chrysia), el Guaraguíto de Sierra (A. striatus), el Cao (C. palmarum) y la Paloma Coronita ( $P$. leucocephala), ver Tabla I. Los principales factores que amenazan estas especies, son la destrucción de hábitat, la casa ilegal y en el caso del Perico y la Cotorra, también la captura para comercio como mascotas (Latta et al., 2006).

Las aves registradas pertenecen a seis gremios alimentarios, siendo los insectívoros (19) los más comunes y abundantes, seguidos de los omnívoros (7), granívoros-frugívoros (5), granívoros (3), carnívoros (3), nectarívoros (3) y frugívoro (1). Algunas especies, pueden ser ubicadas en varios gremios alimentarios; un ejemplo de estas son algunos miembros de las familias Psittacidae y Columbidae, los cuales consumen tanto frutas como semillas (Tabla I).

E1 Hoyo de Pelempito constituye un importante refugio para diferentes especies de aves, tanto migratorias como residentes, especialmente para la Perdiz Grande y la Cotorra, ya que en esta área se registra una población considerable de ambas especies. Igualmente, debido a las condiciones geológicas de la zona, se favorece la presencia de aves insectívoras y granívoras.

\section{AGRADECIMIENTOS}

Al Museo Nacional de Historia Natural "Prof. Eugenio de Jesús Marcano" por el auspicio y financiamiento de este trabajo. A mis compañeros Cristian Marte, Miguel Santiago Núñez, Gabriel de los Santos, Solanlly Carrero y América Sánchez por su ayuda en los viajes de Campo. A Carlos Suriel, por sus sugerencias para la realización de este estudio. 


\section{LITERATURA CITADA}

Banks, R., K. Barker, C. Cicero, J. Dunn, A. Kratter, P. Rasmussen, J. Remsen, J. Rising y D. Stotz. 2005. Forty-Sixth Supplement the American Ornithologists' Union Check-List of North American Birds. The American Ornithologists Union. The Auk, 122(3):1026-1031.

Banks, R., K. Barker, C. Cicero, J. Dunn, A. Kratter, P. Rasmussen, J. Remsen, J. Rising y D. Stotz. 2007. Forty-Eighth Supplement to the American Ornithologists' Union Check-List of North American Birds. The Auk, 124(3):1109-1115.

Banks, R., T. Chesser, C. Cicero, J. Dunn, A. Kratter, I. Lovette, Pamela C. Rasmussen, J. Remsen, J. Rising, D. Stotz, and K. Winker. 2008. Forty-Ninth Supplement to the American Ornithologists' Union Check-list of North American Birds. The American Ornithologists' Union. The Auk, 125(3):758-768.

Banks, R., C. Cicero, J. Dunn, A. Kratter, P. Rasmussen, J. Remsen, J. Rising y D. Stotz. 2006. Forty-Seventh Supplement to the American Ornithologists' Union Check-List of North American Birds. The Auk, 123(3):926-936.

Chesser, T., R. Banks, C. Cicero, J. Dunn, A. Kratter, P. Rasmussen, I. Lovette, J. Remse, J. Rising, D. Stotz y K. Winker. 2009. Fiftieth Supplement to the American Ornithologists' Union Check-list of North American Birds. The American Ornithologists' Union. The Auk,126 (3):705-714. (10).

Chesser, T., R. Banks, K. Barker, C. Cicero, J. Dunn, A. Kratter, I. Lovett e, P. Rasmussen, J. Remsen, J, . Rising, D. Stotz, and K. Winker. 2010. Fifty-First Supplement to the American Ornithologists' Union Check-List of North American Birds. The American Ornithologists' Union. The Auk, 127(3):726-744.

Chesser, T., R. Banks, C. Cicero, J. Dunn, A. Kratter, I. Lovette, A. Navarro-Sig, P. Rasmussen, J. Remsen, J. Rising, D. Stotz y K. Winker. 2014. Fifty-Fifth Supplement to the American Ornithologists 'Union Check-List of North American Birds. The Auk: Ornithological Advances, 131: CSi-CSxv.

Fjeldsa, J. 1999. The impact of human forest disturbance on the endemic avifauna of the Udzungwa Mountains, Tanzania. Bird Conserv. Int., 9 (10):47-62.

Latta, S., C. Rimmer, A. Keth, J. Wiley, H. Raffaele, K. Mcfarland y E. Fernández. 2006. Aves de la República. Dominicana y Haití. Princeton University Press, VII+258 pp. Estados unidos.

Martínez Batlle, J. R. 2002. Sabanas de la República Dominicana: análisis ecodinámico de patrones tipológicos y sus ecotonos. Proyecto de investigación de Doctorado Cambios ambientales y riesgos naturales. Departamento de geografía física y análisis geográfico regional,Universidad de Sevilla.http:/www.geografiafisica.org/wp content/ uploads/2013/02/Tesina-Contacto Bosque-Sabana_reducida.pdf. 9 de julio, 2013.

Perdomo, L., Y. Arias, Y. Léon y D. Wege. 2010. Áreas importantes para la conservación de las aves en la República Dominicana. Grupo Jaragua y el programa IBA-Caribe de Birdlife International: Republica Dominicana. Santo Domingo. República Dominicana.

Ralph, C. J., G. R. Geupel, P. Pyle, T. E. Martin, D. F. DeSante y B. Milá. 1996. Manual de métodos de campo para el monitoreo de aves terrestres. Gen. Tech. Rep. PSW-GTR-159. Albany, CA: Pacific southwest Research Station, Forest services, U. S. Department of agriculture, 46 pp. Marcano, J. 2013. http://mipais.jmarcano.com/. Consultado en fecha 2-XII-2013.

[Recibido: 28 de mayo, 2015. Aceptado para publicación: 22 de agosto, 2015] 